BULLETIN Bulletin hispanique

HispaniQuE Université Michel de Montaigne Bordeaux

120-1 | 2018

Varia

Jean-Pierre Étienvre, Apuntes y despuntes cervantinos Universidad de Alcalá, Instituto Universitario de Investigación Miguel de Cervantes,Alcalá de Henares

Jean Michel Laspéras

\title{
OpenEdition
}

1 Journals

Édition électronique

URL : https://journals.openedition.org/bulletinhispanique/6247

DOI : 10.4000/bulletinhispanique.6247

ISSN : 1775-3821

Éditeur

Presses universitaires de Bordeaux

Édition imprimée

Date de publication : 30 juin 2018

Pagination : 353-358

ISBN : 979-10-300-0298-0

ISSN : 0007-4640

Référence électronique

Jean Michel Laspéras, « Jean-Pierre Étienvre, Apuntes y despuntes cervantinos », Bulletin hispanique [En ligne], 120-1 | 2018, mis en ligne le 30 juin 2018, consulté le 08 janvier 2022. URL : http://

journals.openedition.org/bulletinhispanique/6247 ; DOI : https://doi.org/10.4000/bulletinhispanique. 6247

Ce document a été généré automatiquement le 8 janvier 2022.

Tous droits réservés 


\section{Jean-Pierre Étienvre, Apuntes y despuntes cervantinos}

Universidad de Alcalá, Instituto Universitario de Investigación Miguel de Cervantes,Alcalá de Henares

Jean Michel Laspéras

\section{RÉFÉRENCE}

Jean-Pierre Étienvre, Apuntes y despuntes cervantinos. - Alcalá de Henares : Universidad de Alcalá, Instituto Universitario de Investigación Miguel de Cervantes, 166 p. : Nota de procedencias, Bibliografía, Índice onomástico. ISBN 978-84-16978-00-7.

1 «À la manière de..."; une captatio benevolentice à l'humilité réjouissante inscrit le préambule de cet ouvrage dans la tradition du Siècle d'or. Sur un ton souvent folâtre (fol, belle syllabe pour le Quichotte), Jean-Pierre Étienvre s'y divertit et justifie pour le lecteur les termes du titre et les contenus de neuf communications présentées au fil des ans et dédiées chacune à une ou plusieurs personnes. Il n'ambitionne point l'étude "fondamentale, moins encore axiomatique", de l'œuvre de Cervantès. Apuntes $y$ despuntes cervantinos est écrit dans une langue élégante et châtiée qui ne répugne pas à la liberté de ton pour interpeler sous couvert de modestie la «corporation cervantesque ». Usant du je (comme du jeu) à loisir et satiété, l'auteur n'hésite pas à mettre dans sa poche le lecteur par la prolepse de contenu ou l'horizon d'attente.

Il n'est pas d'exercice intellectuel qui ne soit finalement inutile, opinait - selon Borges Pierre Ménard ${ }^{1}$. Voire! Le lecteur sent dans Apuntes y despuntes cervantinos le plaisir de converser sur le Quichotte et le Persiles avec des publics variés. Et puis la promesse d'une analyse qui se range sous la bannière de la littéralité est un gage de lectures attentives aux moindres frémissements des textes, à l'inattendu des mots et des attitudes, aux jeux et enjeux qui se font et défont sous des formes variées. 
3 Le recueil puise, de l'aveu même de l'auteur, partie de sa substance dans une bibliographie généreuse et complète. Citée avec exigence et probité, elle devrait servir d'exemple à nombre d'émules modernes d'Avellaneda.

4 «Paciencia y barajar: Cervantes, los naipes y la burla» (pp. 15-38) pose la question - non résolue à ce jour - d'un Cervantès joueur, quand bien même la centaine d'entrées du lexique offert à la fin le laisserait penser. Plus important me semble le fait de replacer le thème non dans le cadre d'un prétendu réalisme ou d'un tableau de mœurs que dans celui d'un jeu d'énumérations comme les affectionnait Cervantès. Le paragraphe intitulé "Las flores de Rinconete", ajoute une explication nouvelle du nom de Cortadillo et surtout celle, très documentée et finement analysée, de l'expression caballo de Ginebra. Quant à la problématique du jeu dans l'épisode de Barataria (« Eutrapelia y fiel desengaño »), l'équilibre instable entre eutrapélie et modération légale, entre doctrine et casuistique, met en lumière la (fameuse) ambigüité cervantesque et la remise en jeu du topos «Paciencia y barajar» démontre l'intention satirique du romancier à l'égard du Fiel desengaño de Luque Fajardo et, au-delà, de l'idéal chevaleresque.

5 Le climat de bourle s'illustre ensuite dans un concept égrené au long du Quichotte et que dévoile ce titre : "Figuras del aire en el Quijote » (pp. 39-54). De l'épisode des moulins à vent à Sancho le berné, de Clavileño aux galères de Barcelone, nauséeux ou poudreux, cet élément très structurant est bien mis en perspective par des rapprochements subtils. S'appuyant sur le chapitre soixante-dix de la seconde partie (mort et résurrection d'Altisidora) et toujours soucieux de littéralité, Jean-Pierre Étienvre y réunit trois thèmes, l'air, les diables, les livres. L'apocryphe d'Avellaneda, baudruche que bottent et boutent les diables, est satirisé sur l'air de la bourle et de la farce canine villageoise.

6 La problématique de l'apocryphe se poursuit dans l'article suivant, «La elusión del apócrifo » (pp. 55-67), où sont différenciés avec justesse les concepts de "continuación » apocryphe et de suite authentique. L'auteur situe au chapitre cinquante-neuf le moment de la découverte par Cervantès de la «diablerie " d'Avellaneda et il démonte les mécanismes non d'une vengeance, mais d'un jeu romanesque bien plus subtil et digne : emprunter, "séquestrer » (Martín de Riquer) un personnage de l'apocryphe pour l'introduire dans le roman originel et par là l'authentifier ${ }^{2}$.

7 L'étude « Entre trapa y Troya » (pp. 67-82) concerne les six chapitres de la seconde partie du Quijotte, lors du séjour de don Quichotte et Sancho à Barcelone. Leur analyse linéaire et littérale met en relief un certain nombre de traits pertinents sur les désirs respectifs de Roque Guinard et de don Quichotte. Elle insiste sur la fête qui s'annonce, la SaintJean, et sur la convocation des quatre éléments pour offrir une mise en scène à l'entrée du chevalier et de son écuyer sur la plage de Barcelone. Les festivités dans lesquelles ils sont entraînés n'ont pas été prévues pour se moquer d'eux mais elles donnent lieu à toutes les inquiétudes d'un don Quichotte qui ne s'y sent pas à sa juste place (" en su centro ", p. 73). Évidemment, comme le fait remarquer Jean-Pierre Étienvre, le narrateur, maître de son jeu, fait coïncider le choix de la date, du lieu et de la mise en scène avec la dénonciation de l'apocryphe pour une "réappropriation [par Cervantès] de ses protagonistes dans une atmosphère festive distincte de celle de Saragosse qu'évoque le livre d'Avellaneda. Tout à leur joie de rire sans préjudice d'autrui, les gens réunis autour d'Antonio Moreno ont lu la première partie et même l'apocryphe et ils se réjouissent à plus va jusqu'à ce que la fête soit gâtée par un faiseur de morale et par les 
dames qui tentent de séduire un don Quichotte vite rappelé au souvenir de Dulcinée. C'est son chemin de croix, un vendredi, détail que la critique n'a pas retenu et que l'auteur souligne avec une jouissance non feinte.

8 L'étude passe rapidement sur l'épisode des galères et sur le désir de vengeance du chevalier de la Blanche Lune, alias Sansón Carrasco, pour s'interroger sur Barcelone. Les dernières pages visent non seulement à justifier ce qu'écrivait don Américo, à savoir qu'il s'agissait d'une abstraction, mais à montrer aussi que Cervantès n'était pas dupe et qu'il savait que l'imprimeur d'Avellaneda était Sebastián de Cormellas. Comment expliquer que la seule ville que visite don Quichotte soit Barcelone? Ville d'imprimerie, ville de fête, de comedia - ou de tragédie -, tout s'y joue au point que ces six chapitres apparaissent comme un "Quichotte en miniature ».

Les deux travaux suivants, dont l'ordre aurait pu être inversé, concernent le Persiles. Comme «Los juegos del deseo en el Persiles» (pp. 82-106), ils sont placés sous le signe de la bourle, du jeu et de l'apocryphe. Partant du livre de Jean-Marc Pelorson, El Persiles, ¿novela del deseo?, Jean-Pierre Étienvre note que le désir, même érotique, est dans sa non réalisation même le ressort du roman. Désir de Periandro pour lequel le narrateur utilise ressorts et détours symboliques, alors que celui d'Auristela est tu, même si « son âme est en perpétuel mouvement ». La démonstration conclut à un amour chaste et exemplaire où l'Eros occupe peu de place.

10 Laissant de côté les opinions réductrices ou fondées sur le fait religieux, l'auteur de ces pages propose de considérer que l'œuvre montre les menaces qui pèsent sur l'amour et la manière de surmonter les tribulations du désir, «trabajos del deseo ", afin de mieux les sublimer. Celles que suscite le portrait d'Auristela en offrent le meilleur exemple. Ce thème mériterait une étude à part, ne serait-ce que par la topique de sa représentation et de sa réception.

11 La conclusion, qui s'enracine dans le livre de Pelorson, ajoute qu'il est un désir ultime qui domine la dédicace et le prologue du Persiles : celui de vivre. Vivre pour raconter et, ajouterai-je, celui d'écrire une épopée ou, à l'instar de Shéhérazade, raconter pour vivre.

12 "Trámites y trances en el paratexto del Persiles »: alors que les premiers termes du titre s'éclairent à la fin, il s'agit d'abord de lever les doutes qui ont trait aux démarches légales et à leur contenu. L'auteur pose un certain nombre de questions sur le rôle de la veuve de Cervantès, sur sa hâte à demander les autorisations, sans doute pour des questions économiques liées à la gloire encore vivace du père du Quichotte.

13 L'analyse passe ensuite en revue la question du titre et de ses successives modulations au fil des annonces de l'œuvre pour s'arrêter aux différentes parties ou chapitres du texte du Persiles, et expliquer l'adjectif « septentrional ». Un adjectif qui ne correspond guère aux lieux de l'action et dont il conviendrait d'attribuer l'emploi à un principe éditorial de réclame.

14 L'analyse du paratexte se poursuit par les éloges des contemporains, lesquels vantent le christianisme de l'auteur, "el ingenio cristiano", sans faire allusion au Persiles, tout au moins explicitement. Toujours fidèle au principe de littéralité, Jean-Pierre Étienvre met l'accent sur l'épitaphe et le sonnet dont, implicitement, certains termes renvoient au roman ( “ Caminante, el peregrino / Cervantes... » pour le premier ; « oh caminante », pour le second à la facture "gongorine» imprégnée de relents religieux et moralisateurs). Selon l'auteur de l'article, ce ne fut pas Cervantès, une fois encore, le promoteur de ces 
poèmes, mais sans doute sa veuve ou son éditeur, désireux de présenter l'auteur du Persiles dans une " perspective politiquement [catholiquement] correcte ».

La dernière partie de ces pages et leur conclusion s'imposent à mes yeux comme un aute moment fort de Apuntes y despuntes cervantinos en raison de la réflexion sur le rapport - ou plutôt son absence - entre les pièces paratextuelles et la narration. Textes autonomes qui, s'ils clôturent le livre, ne s'en ouvrent pas moins sur un futur qui concerne des œuvres à venir ou non achevées (Semanas del jardín, El Bernardo). Du prologue, délaissant les gloses qui en ont été produites, Jean-Pierre Étienvre choisit la traduction de l'oxymore "manco sano " proposée par Jean-Marc Pelorson pour offrir plusieurs possibilités d'interprétation, dont celles du «famoso todo » et de l'expression «[...] presto contentos en la otra vida ». Et d'en questionner l'adverbe pour suggérer que la satisfaction viendrait des dits, des ris, des réjouissances entre amis, alors que l'on pourrait s'attendre, au vu des circonstances, à des préoccupations eschatologiques. Ainsi se pose non seulement le rapport à l'ami-lecteur, mais encore et surtout au fait religieux qui mériterait pour l'œuvre de Cervantès une mise au point sereine.

Réception et interprétation : ainsi s'ouvre le débat développé dans «Cervantes al margen de los clásios en la España del siglo XVII» (pp. 107-120) à propos de la désaffection et de l'indifférence dont eurent à pâtir le Quichotte et son auteur. On connaît les difficultés de Francisco de Robles pour écouler la seconde partie du roman ${ }^{3}$, de même que le dépit et l'amertume de Cervantès devant l'insuccès de son théâtre. Se saisissant de la curieuse anecdote qui occupe tout le prologue du Persiles, Jean-Pierre Étienvre perçoit le discours comme un "amertume chrématistique » et lui donne comme prolongement une réflexion très documentée sur la réception du Quichotte. Outre le jugement moral de Tirso de Molina à propos des livres de chevalerie et celui, peu amène, de Lope de Vega, il ressort que les contemporains espagnols en firent un livre de divertissement où le rire le disputait à la satire. Mais la vis comica s'était banalisée, les figures des deux héros s'étaient vulgarisées, "carnavalisées " même, au point d'être dissociées de la création cervantesque. Et puis la seconde partie du roman, plus complexe, moins encline à la tradition burlesque, ne répondait plus aux attentes d'un public attiré par d'autres formes littéraires.

Enfin Azorín vint. Jean-Pierre Étienvre note que de façon anachronique l'auteur de Clásicos y modernos n'entendait pas que les hommes du XVII ${ }^{\mathrm{e}}$ n'eussent pas rangé Cervantès sous la bannière des "classiques ». Et l'article de montrer que s'il est des exceptions comme Valdivielso, le cas de Gracián est assez décevant. Heureusement, dans cet océan d'indifférence et de mépris, il convient de saluer, à la fin du même siècle, un Nicolás Antonio, lequel ouvre la voie à Mayans, dont nous pouvons partager la sûreté du jugement. Cité fort à propos, il éclaire l'ostracisme dont a souffert Cervantès en tant qu'auteur et en tant que «classique». Homme du XVI ${ }^{e}$, il fut malheureusement un écrivain du XVII ${ }^{e}$; telle est la conclusion que suggèrent ces pages très bien documentées.

Les deux derniers articles dépassent le strict cadre textuel de l'œuvre de Cervantès pour faire une place l'un à Michel Foucault, l'autre à Maurice Molho. Dans «El Quijote de Michel Foucault: un "teatro lúdico" entre polémicas» (pp.121-131), Jean-Pierre Étienvre se place au-dessus de la mêlée, c'est-à-dire sans entrer dans la polémique suscitée chez les hispanistes par l'analyse de Foucault. Il s'intéresse d'abord à la découverte du Quichotte par le philosophe dans une archéologie de son savoir "quichottesque », puis il résume ses échanges enfiévrés avec Jean-Marc Pelorson qui 
lui reproche, dans un numéro de La Pensée (1970), d'ignorer les travaux des historiens et des hispanistes. Un seconde phase de l'écrit montre qu'avec le temps et l'apaisement certaines idées du philosophe furent peu à peu acceptées. La conclusion fait une large place à une longue citation où Foucault évoque la grande comédie des signes et des choses, le théâtre ludique qu'était pour lui le Quichotte.

Quant à la fameuse phrase finale du prologue du Persiles, «Adiós gracias; adiós donaires; adiós regocijados amigos (la loca amenidad de Maurice Molho)» (pp. 133-143), il s'agit d'un hommage personnel qui rappellera des souvenirs à ceux qui ont connu Maurice Molho ou qui ont été ses disciples. D'entre les réflexions et les marques d'admiration de JeanPierre Étienvre, cette remarque: «[...] había que aceptar el peligro de las hipótesis impertinentes para tener acceso a un posible descubrimiento " (p. 135). Comme l'avouaient d'aucuns de la "confrérie" qui n'étaient pas de ses amis, il faisait quand même «bouger les choses". Et l'on retiendra son questionnement sur le "Garcilaso sans commentaire » ou sur le détour par Lorette de Tomás Rodaja, interrogations auxquelles pourrait s'ajouter la question de l'hydropisie dans L'illustre souillon et dans ce même prologue.

Apuntes y despuntes cervantinos est un livre érudit et suggestif où le concept de jeu le dispute à l'ironie et au rire. Le ton enjoué qui préside à l'évocation de la confrérie des «locos amenos » baigne le recueil et irrigue le réseau de sens que fait surgir Jean-Pierre Étienvre, du don Quichotte au Persiles. Il accompagne le lecteur vers cette conception de la littérature où la gravité fait jeu égal avec la satire et la bourle. En dépit de quelques redites (pp. 39, 69) et de citations répétées, les analyses souvent conduites hors des sentiers battus apportent un air nouveau - sujet oblige - et, l'humilité de l'auteur dûtelle en souffrir, une pierre à l'édifice critique.

\section{NOTES}

1. Cité p. 121.

2. D'ordinaire l'adjectif apocryphe qualifie un auteur ou une œuvre. Or, dans cette citation, il renvoie au personnage : «[...] el valeroso don Quijote de la Mancha, no el falso, o el ficticio, no el apócrifo que en falsas historias estos días nos han mostrado » (II, 61, p. 1235 ).

3. Erreur bibliographique et oubli sans doute, il restait dans la boutique de l'éditeur Robles 367 exemplaires de la seconde partie et non 366, comme il est écrit ici. L'inventaire, souvent signalé par Francisco Rico (1998, CCXXXVIII-CCXXXIX), a été publié par mes soins ( El fondo de librería de Francisco de Robles, editor de Cervantes" Cuadernos Bibliográficos, XXXVIII (1979), pp. 107-138). 


\section{AUTEURS}

JEAN MICHEL LASPÉRAS

Université Provence 